\title{
Target uncertainty does not lead to more distraction by singletons: Intertrial priming does
}

\author{
YAÏR PINTO, CHRISTIAN N. L. OLIVERS, and JAN THEEUWES \\ Vrije Universiteit, Amsterdam, The Netherlands
}

\begin{abstract}
In two experiments, we examined why a singleton distractor has a stronger interfering effect in visual search when the target identity is uncertain. When participants searched for a shape, a color singleton distractor had a larger slowing effect in a mixed block, in which the target shape could change from trial to trial, than in a pure block, in which the target shape remained the same. Importantly, this increased singleton distractor effect could be traced back entirely to intertrial priming, since the increased costs occurred only on trials in which the target and the singleton distractor swapped identity (Experiment 1, allowing for priming between targets and singleton distractors) or on trials in which the target alone changed identity while the singleton distractor remained constant (Experiment 2, allowing for priming between targets only). This suggests that target uncertainty itself does not lead to strategic changes in the attentional selection of singletons. Instead, selection is affected by relatively automatic priming mechanisms that may be enhanced by competition for attention.
\end{abstract}

In our everyday lives, we are presented with a wide variety of visual stimuli. For adaptive behavior, it is important to select both objects relevant to our goals and objects that might not be directly relevant but have an intrinsic importance. A key discussion in attention research is the interaction between these goals and the intrinsic qualities of objects in the visual field. The discussion revolves around the concept of attentional capture, defined as the involuntarily drawing of attention.

According to the bottom-up view, objects carrying a unique feature may automatically capture attention as a result of their relative salience (Theeuwes, 1991). These objects are often referred to as singletons, since they are unique on a certain dimension (Pashler, 1988). For instance, in Theeuwes's (1991) task, people searched for either a diamond among circles or a circle among diamonds. These display types were randomly mixed so that the participants would not know beforehand which shape to look for. Although the task was to look for the odd shape, reaction times (RTs) were about $150 \mathrm{msec}$ longer when a differently colored singleton distractor was also present. Since the singleton was completely irrelevant to the task, Theeuwes (1991) concluded that it captured attention automatically.

In a subsequent series of experiments, Theeuwes (1992) replicated his earlier findings, except that now, throughout a block, the target remained the same - that is, within one

This research was funded by a grant from NWO (Netherlands Organization for Scientific Research), Grant 400-03-008 to J.T., and Grant 451-02-117 to C.N.L.O. We thank Richard Abrams and three anonymous reviewers for their constructive comments on an earlier version. Correspondence concerning this article should be addressed to Y. Pinto, Department of Cognitive Psychology, Vrije Universiteit, Van der Boechhorststraat 1, 1081 BT Amsterdam, The Netherlands (e-mail: y.pinto@) psy.vu.nl). block, the target was always a diamond among circles, and within another block, it was always a circle among diamonds - while a singleton distractor could be present. The participants were still distracted by the singleton distractor, but now it had a slowing effect of only about $20 \mathrm{msec}$ (as compared with the $150 \mathrm{msec}$ found by Theeuwes, 1991).

These findings appear to be difficult to explain from a pure bottom-up view. Note that the stimuli were exactly the same in both studies, the only difference being that, in Theeuwes (1991), target types were mixed and, thus, the participants did not know beforehand what the target would be. Instead, Theeuwes's $(1991,1992)$ findings suggest that attentional capture by the singleton is task dependent, since target preknowledge influences the distracting effect of the singleton. This seems more in accordance with a top-down view of attentional capture (see also Folk, Remington, \& Johnston, 1992; Yantis \& Egeth, 1999). Presumably, when the target is uncertain, participants adopt a less restrictive attentional set, to accommodate the differences in target appearance. This less restrictive attentional set then makes the participants more susceptible to singleton distractors. This notion of a more or less restrictive attentional set is in accordance with what Bacon and Egeth (1994) referred to as the difference between the feature search mode and the singleton detection mode of attention. Bacon and Egeth also found that an irrelevant singleton distractor interfered with search, but only if the target itself was also a singleton-namely, the odd shape. In contrast, when trials were intermixed with trials on which there was no single unique shape target, the singleton distractor lost its interfering effect. Bacon and Egeth argued that when the target itself is a unique singleton, as was the case in Theeuwes's $(1991,1992)$ experiments, it may be beneficial to adopt a singleton search strategy, in which the observer looks for "any" singleton. This would then also include the singleton distractor. In 
contrast, when the target is not unique, the observer may adopt a feature search strategy, in which selection is confined to a specific feature to the exclusion of other singletons. Important for the present study, Bacon and Egeth argued that people are forced to adopt a singleton detection strategy when the target is uncertain, since they do not know the specific features of the target. Thus, the wider attentional set associated with singleton detection mode may explain the increased singleton distraction found in the mixed condition in Theeuwes (1991).

However, there is an alternative explanation, more in line with Theeuwes's original stimulus-driven account of events. According to this view, the differential singleton distractor effects found by Theeuwes $(1991,1992)$ can be explained by assuming a role for intertrial priming. According to the intertrial priming account, previous trials have an automatic effect on the current trial: Features associated with the target are facilitated, whereas features associated with a distractor are inhibited (Maljkovic \& Nakayama, 1994, 2000; Müller, Heller, \& Ziegler, 1995; Olivers \& Humphreys, 2003; see also the literature on negative priming for similar carryover effects in nonsearch tasks: e.g., Tipper, 1985). This relative activation and inhibition automatically carries over to the next trial. In a mixed block, then, features of a distractor on one trial can become features of the target on the next trial, resulting in a relative slowing of target detection. Conversely, features of the target on one trial can become features of the singleton distractor on the next, possibly leading to a relative boost of its salience, due to carried-over activation. For instance, in Theeuwes's (1991) study, on one trial, the target might be a circle, whereas the singleton distractor might be a diamond. On the next trial, this might be reversed, so that the target (now a diamond) receives carried-over inhibition from the previous singleton distractor, whereas the singleton distractor (now a circle) receives facilitation from the previous target. This caused larger interference of the singleton distractor and less efficient search for the target. In a pure block, features of a distractor on one trial never became features of the target on the next trial, or vice versa. Therefore, target features could be maximally activated from trial to trial, whereas distractor features could be maximally inhibited, leading to a reduced singleton distractor effect.

The purpose of the present study was, first to replicate Theeuwes's $(1991,1992)$ results within a single experiment and to determine whether people are, indeed, more distracted by an irrelevant color singleton when target types are mixed. Second, we analyzed intertrial effects, to determine whether the increased singleton distractor effect can at least partially be explained by intertrial priming. In the mixed condition in Experiment 1, the target shape and the nontarget shape could switch from trial to trial, and on an intertrial priming account, priming could operate between targets alone, between nontargets alone, and between targets and nontargets. In the mixed condition in Experiment 2, the target shape could change identity, whereas the nontarget shape remained the same throughout a block, not allowing for priming between targets and nontargets. If the priming between targets and singleton distractors is an important contributor to the increased singleton distractor effect, we should see this effect reduced in Experiment 2. But if priming between targets alone is also important, we should see at least some residual intertrial effects re-emerge.

\section{EXPERIMENT 1}

In the first experiment, we presented participants with three conditions. In the pure diamond condition, the participants looked for a diamond among circles, in the pure circle condition, the participants looked for a circle among diamonds. In the mixed condition, the participants looked for either a diamond among circles or a circle among diamonds. Thus, the task was always to look for an odd shape, but in the pure conditions the participants knew beforehand what the target would be, whereas in the mixed condition they did not. The participants performed a so-called compound search task, in which they responded to the line segment inside the target.

A second important manipulation involved the presence of a singleton distractor. On the basis of Theeuwes (1991, 1992), we expected an irrelevant color singleton to result in a larger slowing of RTs in the mixed condition than in the pure conditions. Importantly, to assess whether this increased singleton distractor effect was due to an overall change in search strategy (i.e., the adoption of a broader attentional set), to intertrial priming, or to both, we looked at intertrial relationships within the mixed block. Switch trials were defined as trials on which the target differed from the target on the previous trial, and same trials were defined as those on which the target was the same as that on the previous trial (see Figure 1 for example displays of a same and a switch trial). Note that on switch trials, the singleton distractor changed identity too. If intertrial priming plays a role in causing the increased singleton distractor effect, it would be expected that RT costs resulting from the presence of a singleton distractor would be larger on switch than on same trials, since on switch trials the singleton distractor might receive activation carried over from the previous target shape, whereas the target might receive inhibition carried over from the previous singleton distractor shape. If, in addition to any intertrial priming, there is also an overall change in search strategy between pure and mixed conditions, we would expect an increased singleton distractor effect not only on switch trials, but also on same trials.

\section{Method}

Participants. Eight participants, ranging in age from 21 to 31 years (average, 24.3 years) took part as paid volunteers. All the participants completed all of the conditions. All had normal or correctedto-normal vision.

Apparatus and Stimuli. The experiment was conducted on a computer with a Pentium II processor, a 17-in. monitor, and a standard QWERTY keyboard. The software package E-Prime was used for the layout and timing of the experimental trials. The stimulus field consisted of nine elements equally spaced on an imaginary circle (diameter, $11^{\circ}$ ), around the fixation point (diameter, $0.32^{\circ}$ ). The ele- 


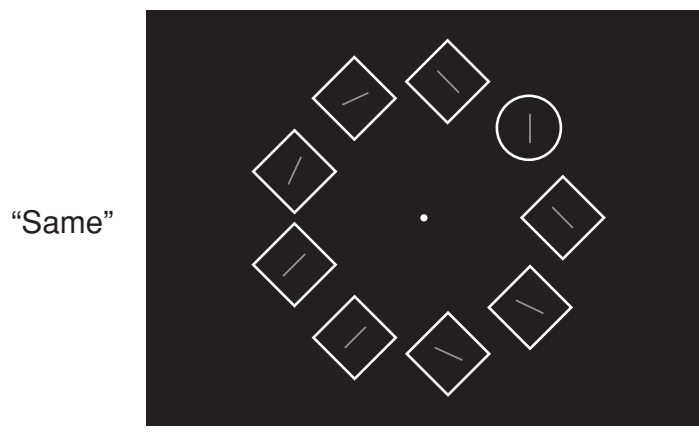

Trial $n$

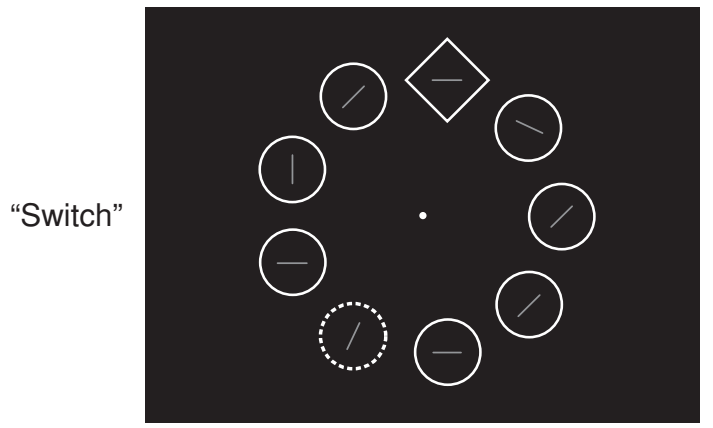

Trial $n$

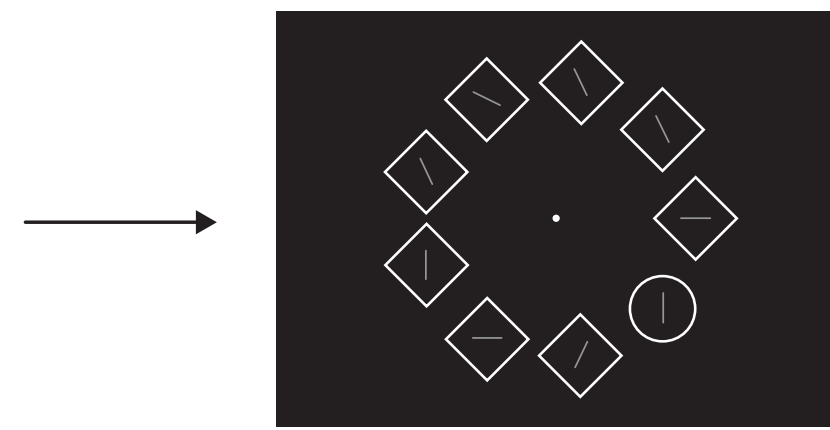

Trial $n+1$

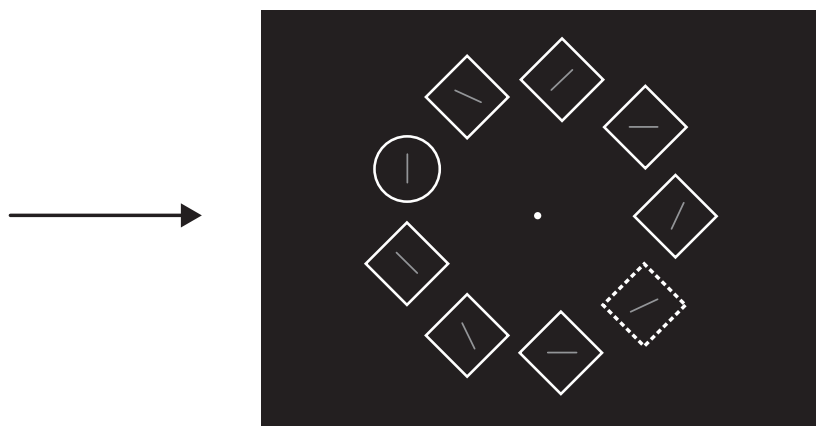

Trial $n+1$

Figure 1. Example of a stimulus display in Experiment 1. The top panel depicts a same trial without a singleton distractor; the bottom panel depicts a switch trial with a singleton distractor. The target was always the odd shape (circle or diamond), and participants responded to the orientation of the line segment inside it. All the items were gray except for the singleton distractor, which deviated in color (red or green, as indicated here by a dotted outline).

ments were open diamonds (diameter, $3.5^{\circ}$ ) and open circles (diameter, $\left.2.8^{\circ}\right)$ that were red (CIE $x$-, $y$-coordinates: $\left..607, .351\right)$, green $(.245, .577)$, or gray $(.259, .314)$. All colors were equiluminant $\left(7 \mathrm{~cd} / \mathrm{m}^{2}\right)$, as measured with a Tektronix photometer. The fixation spot was white $\left(51.2 \mathrm{~cd} / \mathrm{m}^{2}\right)$, and the background black $\left(0 \mathrm{~cd} / \mathrm{m}^{2}\right)$. All the elements contained gray line segments (diameter, $\left.1.2^{\circ}\right)$, that were randomly tilted horizontally, vertically, or $22.5^{\circ}$ to either side of the horizontal or vertical. In the target element, the line segment was oriented either horizontally or vertically. The position of the target element was randomly chosen from the nine possible positions.

Procedure. The participants sat approximately $90 \mathrm{~cm}$ from the monitor, with their fingers resting on the " $\mathrm{z}$ " and " $\mathrm{m}$ " keys, which were used as the response buttons. The experiment consisted of five clusters of six blocks, each containing 16 trials. The order of the blocks within a cluster was random. In each cluster there were three singleton distractor absent blocks, in which no singleton distractor was present on any trial. In the circle condition, the participants looked for a gray circle among gray diamonds. In the diamond condition, the participants looked for a gray diamond among gray circles. In the mixed condition, the participants looked either for a gray diamond among gray circles or for a gray circle among gray diamonds. Targets were randomly mixed so that the participants would not know beforehand what the target element would be. In the singleton distractor present condition, the stimuli were the same as those in the singleton distractor absent condition, the only difference being that one of the nontarget elements was replaced by a uniquely colored singleton distractor element on every trial. Singleton distractor presence was blocked for direct compatibility with Theeuwes (1991, 1992). In the circle condition, the singleton distractor was a red or a green diamond. In the diamond condition, the singleton distractor was a red or a green circle. In the mixed condition, the singleton distractor depended on the target: a red or a green diamond when the target was a circle, and a red or a green circle when the target was a diamond. The position of the singleton distractor was randomly determined out of the eight possible positions left, one position being filled by the target element. In every condition, the participants were instructed to determine the orientation of the line segment in the target element. They pressed " $z$ " for vertical and " $m$ " for horizontal segments. The task was assumed to require focal attention to be directed to the target element. Before every block, there appeared a text on the screen instructing the participants which was the type of target: circle, diamond, or mixed. The participants were told to use this information. At the start of the experiment, the participants were told that a singleton distractor could be present during trials but that this singleton distractor was irrelevant to the task. The participants were instructed that both speed and accuracy were important. The first cluster of blocks was disregarded as practice. The other four clusters were included in the analyses. The experiment took approximately $30 \mathrm{~min}$ and was performed without breaks.

\section{Results and Discussion}

Error percentages were low overall (see Table 1), and an ANOVA revealed no significant effects. We will therefore concentrate on the mean RTs for the correct trials.

Overall RTs. Trials on which RTs were greater than $3,000 \mathrm{msec}$ were excluded from analysis, resulting in a loss of fewer than $1 \%$ of the trials. An initial ANOVA revealed that the participants found it easier overall to look for a circle among diamonds than for a diamond among circles $\left[F(1,7)=31.7, M S_{\mathrm{e}}=1,406.2, p=.001\right]$. How- 
Table 1

Average Percentages of Errors for the Different Conditions in Experiments 1 and 2

\begin{tabular}{lcc}
\multicolumn{3}{c}{ in Experiments 1 and 2 } \\
\hline Condition & $\begin{array}{c}\text { Singleton } \\
\text { Distractor Absent }\end{array}$ & $\begin{array}{c}\text { Singleton } \\
\text { Distractor Present }\end{array}$ \\
\hline Circle & Experiment 1 \\
Diamond & 4.9 & 3.1 \\
Mixed & 3.7 & 3.7 \\
& 4.7 & 3.7 \\
Circle & Experiment 2 & \\
Diamond & 3.6 & 4.7 \\
Mixed & 4.9 & 5.3 \\
\hline
\end{tabular}

ever, since there was no interaction between singleton distractor presence and target shape $(F<1)$, we averaged the circle and diamond conditions together to form the pure condition for the following analyses. Figure 2 shows the overall RTs in pure and mixed conditions (for the mixed conditions, the average mixed condition and the mixed condition split into switch and same trials are shown) when a singleton distractor was either present or absent. A two-way ANOVA on mean RT for each participant, with condition (mixed or pure) and singleton distractor (present or absent) as factors, revealed a main effect for condition $\left[F(1,7)=131.6, M S_{\mathrm{e}}=3,352.1, p<.001\right]$, a main effect for singleton distractor $\left[F(1,7)=79.9, M S_{\mathrm{e}}=834.8, p<\right.$ $.001]$, and a significant interaction $\left[F(1,7)=32.4, M S_{\mathrm{e}}=\right.$ $601.2, p=.001]$. The overall pattern of results is very similar to that in Theeuwes $(1991,1992)$. The participants were significantly slower in the mixed condition than in the pure condition, and they were significantly slower when a singleton distractor was present than when it was absent. Finally, as can be seen in Figure 2, a singleton distractor

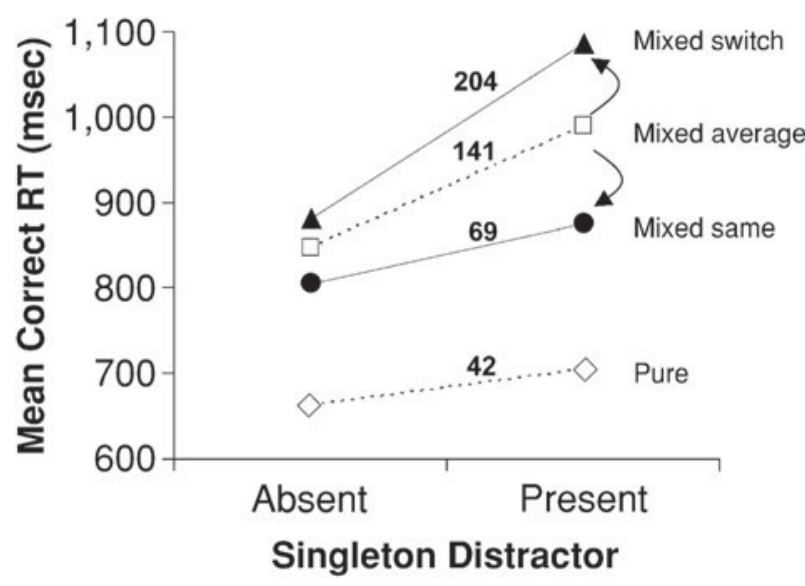

Figure 2. Mean reaction time (RT) results of Experiment 1 as a function of singleton distractor presence. Open symbols reflect the overall mean RTs for the pure and mixed conditions. Filled symbols reflect the mean RTs in the mixed condition, broken down for the different intertrial relationships (same vs. switch). Adjacent to each line, the numerical value (in milliseconds) of the singleton distractor effect is given. had a significantly greater effect in the mixed condition (a cost of $141 \mathrm{msec}$ ) than in the pure condition (a cost of $42 \mathrm{msec}$; both costs are comparable to those reported by Theeuwes, who found 150 and $20 \mathrm{msec}$, respectively). Student $t$ tests revealed that all pairwise comparisons were significant (all $t \mathrm{~s}>4.4$, all $p \mathrm{~s}<.005$ ).

Intertrial effects. To assess the source of the increased singleton distractor effect in the mixed condition, a twoway ANOVA was performed on RTs for trial $n$, as a function of trial $n-1$. One main factor was intertrial relationship: A trial could contain either the same target as the previous trial (same trials) or a different target (switch trials). The other factor was the presence of a singleton distractor (present or absent; see Figure 2 for a graphical depiction of the findings). Main effects were found for intertrial relationship $\left[F(1,7)=79.0, M S_{\mathrm{e}}=2,096.1\right.$, $p<.001]$ and for singleton distractor presence $[F(1,7)=$ $\left.79.4, M S_{\mathrm{e}}=1,873.1, p<.001\right]$. The interaction was also significant $\left[F(1,7)=21.5, M S_{\mathrm{e}}=1,679.6, p<.005\right]$. The participants were significantly slower on switch trials than on same trials. They were also significantly slower when a singleton distractor was present than when it was absent. Furthermore, the significant interaction reflects that the participants were slowed more by the singleton distractor on switch trials (a cost of $204 \mathrm{msec}$ ) than on same trials (a cost of $69 \mathrm{msec}$ ). All pairwise comparisons were significant, as revealed by $t$ tests (all $t \mathrm{~s}>3.6$, all $p$ s <.01). Apparently, then, intertrial effects are a major contributor to the differential singleton distractor effect. Performance suffered from a singleton distractor more on switch trials than on same trials. This result is in accordance with the notion that priming plays an important role: The switching of the target and the singleton distractor identities causes a relative decrease of target activation and a relative increase of singleton distractor activation. It is also in accordance with previous work demonstrating the contribution of intertrial effects to visual search (Maljkovic \& Nakayama, 1994; Müller et al., 1995; Olivers \& Humphreys, 2003).

In addition to the target and singleton distractor interchanging shape, the singleton distractors themselves could change color (from red to green). This enabled us to see whether priming between singleton distractor colors also played a role. Although there was indeed a small cost for a color change $(6 \mathrm{msec}$, averaged across pure and mixed conditions), it was far from significant and did not interact with condition (mixed or pure; all $F_{\mathrm{s}}<1$ ).

An important question is whether the intertrial effects were the only contributors to the increased singleton distractor effect in the mixed condition or whether there were any additional, overall differences. Such overall differences between the mixed and the pure conditions, if present, might provide evidence that differences in topdown strategic settings, such as the overall widening of the attentional set to incorporate the uncertainty of the target, affect attentional capture. One way to assess such potential strategic effects is to look only at same trials in the mixed condition and compare them with the pure condition. After all, the pure condition consists only of 
same trials. If there are any strategic overall changes that lead to increased singleton distractor interference, then, when comparing the mixed condition with the pure condition, we should expect an increased singleton distractor effect not only on switch trials, but also on same trials. In fact, it turned out that the costs associated with the singleton distractor were somewhat higher for the same trials in the mixed condition $(69 \mathrm{msec})$ than in the pure condition (42 msec). However, this difference was not significant $[t(7)<1.18, p>.25]$. Moreover, the increase in the mixed condition may actually not have been due to an overall strategy change but to remnants of priming effects dating from switches occurring more than one trial back. Indeed, the singleton distractor costs in the mixed condition were further reduced when there was more than one consecutive same trial ( $58 \mathrm{msec}$ for two and $44 \mathrm{msec}$ for three consecutive same trials), which suggests that the priming effects require some time/trials to build up to their maximum level. Together, the findings imply that strategic differences between the pure and the mixed conditions play no role in causing the differential singleton distractor effect. This effect seems to be due solely to intertrial priming.

\section{EXPERIMENT 2}

In the mixed condition in Experiment 1, the target and the singleton distractor shape could swap identity. This allowed priming to occur directly between the target and the singleton distractor, as well as between the targets themselves and the singleton distractors themselves. To assess the effects of target uncertainty on singleton capture in more detail, Experiment 2 eliminated priming between targets and singleton distractors. The target was again uncertain (i.e., it could be a circle or a diamond), but now the singleton distractor remained a constant heptagon (see Figure 3 for an example display). This way, the singleton distractor shape could not be primed by the target shape, or vice versa. If increased singleton distractor effects are due to changes in overall strategy related to target uncertainty, we should see these increased singleton distractor effects prevail here (since the target was still uncertain). In contrast, if priming between targets and singleton distractors plays a major or even the only role, the differential singleton distractor costs between mixed and pure conditions should be much reduced or even disappear.

A factor that remains is the priming between targets themselves. Priming between targets may also contribute to differential singleton distractor effects, since the different activations carried over from the previous trial may give the target either more or less of an advantage, relative to the singleton distractor. The second aim of Experiment 2 was, therefore, to assess whether there were any remaining intertrial effects, which, if present, might then be attributed to priming between targets.

\section{Method}

Participants. Twenty participants, ranging in age from 18 to 28 years (average, 20.5 years) took part as paid volunteers. All

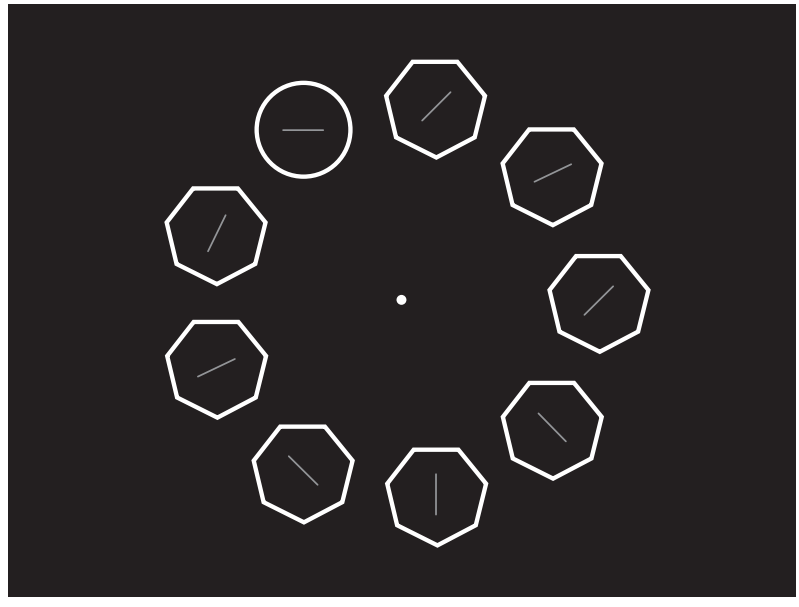

Figure 3. Example display in Experiment 2, which contained heptagons as nontargets. In this particular display, the target was a circle, but it also could be a diamond.

the participants completed all of the conditions. All had normal or corrected-to-normal vision.

Apparatus, Stimuli, and Procedure. The apparatus, stimuli, and procedure were the same as those in Experiment 1, except for the following changes. All nontargets were now heptagons (diameter, $\left.2.9^{\circ}\right)$. In the singleton distractor present condition, one of the heptagons changed color (to green or red), to become the singleton distractor. The target was a circle or a diamond. As before, within the pure condition the target did not change, whereas in the mixed condition the target could vary from trial to trial.

\section{Results and Discussion}

Error percentages were low overall (see Table 1), and an ANOVA revealed no significant effects. We will therefore concentrate on the mean RTs of correct trials.

Overall RTs. Trials on which RTs were greater than 3,000 msec were excluded from analysis, resulting in a loss of fewer than $1 \%$ of the trials. Figure 4 shows the main RT results. There were no significant effects related to the different target shapes $(F \mathrm{~s}<2, p \mathrm{~s}>.15)$, so we again averaged the circle and the diamond conditions together, to form the pure condition for the remaining analyses. A two-way ANOVA on mean RT for each participant, with condition (mixed or pure) and singleton distractor (present or absent) as factors, revealed a main effect of condition $[F(1,19)=$ 107.1, $\left.M S_{\mathrm{e}}=5,823.8, p<.001\right]$, a main effect for singleton distractor $\left[F\left(1,19=24.3, M S_{\mathrm{e}}=1,405.1, p<\right.\right.$ $.001]$, and a trend for the interaction $[F(1,19)=3.13$, $\left.M S_{\mathrm{e}}=1,135.2, p<.1\right]$. The participants were significantly slower overall in the mixed condition than in the pure condition, and they were significantly slower when a singleton distractor was present than when it was absent. Finally, although not significant, there was a tendency toward an increased singleton distractor effect in the mixed condition (cost, $55 \mathrm{msec}$ ), relative to the pure condition (28 $\mathrm{msec})$. All pairwise comparisons were significant, as was revealed by $t$ tests (all $t \mathrm{~s}>3.2$, all $p \mathrm{~s}<.005$ ). The results support the idea that the increased singleton distractor costs in the mixed condition in Experiment 1 were, 


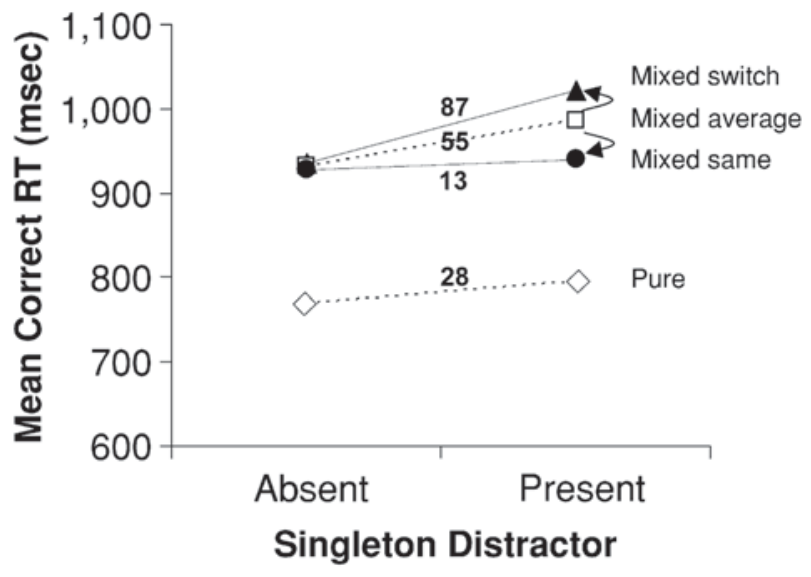

Figure 4. Mean reaction time (RT) results of Experiment 2 as a function of singleton distractor presence. Open symbols reflect the overall mean RTs for the pure and mixed conditions. Filled symbols reflect the mean RTs in the mixed condition, broken down for the different intertrial relationships (same vs. switch). Adjacent to each line, the numerical value (in milliseconds) of the singleton distractor effect is given.

to a large extent, caused by priming between targets and singleton distractors. In Experiment 2, priming between targets and singleton distractors was eliminated, and the differential singleton distractor effect was reduced to a nonsignificant trend (despite the fact that this experiment had more participants). The results also argue against the possibility that increased singleton distractor costs were caused by a change in overall search strategy associated with the uncertainty of the target identity. In Experiment 2, the identity of the target was as uncertain as in Experiment 1, yet the effect of the singleton distractor was much reduced.

Intertrial effects. Even though the differential singleton distractor effect was much reduced (as was expected on the basis of priming between targets and singleton distractors being eliminated), there was still a trend toward increased singleton distractor interference in the mixed condition. To see whether any of these costs might be explained by an overall strategy change, intertrial effects were calculated in the same way as in Experiment 1 (Figure 4 presents a graphical depiction of the findings). Main effects were found for intertrial relationship $[F(1,19)=$ 7.58, $\left.M S_{\mathrm{e}}=5,682.6, p<.05\right]$ and for singleton distractor presence $\left[F(1,19)=13.4, M S_{\mathrm{e}}=3,742.1, p<.005\right]$. Importantly, the interaction was also significant $[F(1,19)=$ 7.48, $\left.M S_{\mathrm{e}}=3,632.3, p<.05\right]$. The participants were significantly slower on switch trials than on same trials. They were also significantly slower when a singleton distractor was present than when it was absent. Furthermore, the interaction reflects the fact that the participants were more distracted by the singleton distractor on switch trials than on same trials, with respective costs of 87 and $13 \mathrm{msec}$. Student $t$ tests showed a significant effect for intertrial relationship for singleton distractor present trials, but not for singleton distractor absent trials [present, $t(19)=3.69, p<.005$; absent, $t(19)=0.46, p>.5]$. To assess whether there were any residual overall increases in singleton distractor costs, the same trials in the mixed condition were compared with the trials in the pure condition (which contained only same trials). There was no significant difference between these trials $(p>.45)$, and if anything, the singleton distractor costs in the mixed condition were smaller than those in the pure condition. Thus, whatever trend there was toward a differential singleton distractor effect, it can again be traced back entirely to intertrial differences, leaving no room for overall strategy changes.

The fact that the singleton distractor interfered more on switch than on same trials is interesting, because the differential intertrial priming mechanisms thought to apply here are limited to only those operating between the targets (since we eliminated the relationship between targets and singleton distractors), and the same targets were used whether a singleton distractor was present or not. This raises the possibility that intertrial priming effects are enhanced by the presence of a singleton distractor, even if the singleton distractor itself is not primed (and does not, in turn, prime the target). The presence of a singleton distractor increases the competition for selection, and this may require more attentional weight to be applied to the target, leading to stronger activation on the subsequent trial.

As in Experiment 1, the singleton distractor, when present, could be either red or green. This allowed us to assess priming between the singleton distractors themselves. On average, across the singleton distractor present blocks of the mixed and pure conditions, the costs of a color change were now a significant $26 \mathrm{msec}[F(1,19)=$ $\left.8.44, M S_{\mathrm{e}}=1,651.7, p<.01\right]$. There were no interactions with condition $(F<1)$. Thus, distractor-distractor priming also appeared to operate, but its magnitude was not affected by mixing target types.

Finally, we should mention that even though the increased singleton distractor costs in the mixed condition were not due to an overall effect, the RTs as a whole were overall increased in the mixed condition relative to the pure condition. We will return to this in the General Discussion section.

\section{GENERAL DISCUSSION}

The goal of our study was to examine how target uncertainty affects attentional capture by irrelevant singleton distractors. We presented observers with pure blocks, throughout which the shape of the targets and singleton distractors stayed the same, and mixed blocks, in which the shape of the targets and singleton distractors switched randomly from trial to trial. Confirming earlier findings (Theeuwes, 1991, 1992), we found that the costs associated with the presence of a singleton distractor were increased in the mixed condition. By comparing the singleton distractor effect on switch and same trials in the mixed condition with the singleton distractor effect in the pure condition, we assessed the relative contributions of intertrial and overall effects to this increase. In both 
Experiments 1 and 2, the differential singleton distractor effect could be explained solely by intertrial effects, with negligible to nonexistent residual overall increases in singleton distractor effects. In Experiment 1, the target and the singleton distractor could swap identity, allowing for priming between the target and the singleton distractor. In Experiment 2, the singleton distractor and the target shapes were always different, and priming between targets and singleton distractors was therefore eliminated. We still found an interaction between singleton distractor presence and intertrial effects, indicating that priming between targets alone also contributes to the efficiency with which a target is selected and a singleton distractor is ignored. Nevertheless, the differential singleton distractor costs were strongly reduced, relative to Experiment 1, indicating that the priming between targets and singleton distractors in Experiment 1 was a major cause of the increased singleton distractor effects.

\section{Intertrial Priming}

An important question is whether the intertrial effects found here are the result of automatic processes or whether they may be traced back to online top-down strategy changes. So far, we have referred to these effects as stemming from priming, which implies an automatic process. Support for this comes from Maljkovic and Nakayama (1994; see also Hillstrom, 2000; Olivers \& Humphreys, 2003), who showed that the intertrial effects can go back as far as eight trials. It is unlikely that observers consciously base their search strategy on these distant trials. Indeed, Maljkovic and Nakayama (2000) have shown that observers hardly remember what they saw on the immediately preceding trial. A second argument against conscious switching of search strategy comes from another experiment from Maljkovic and Nakayama (1994), in which observers could explicitly expect a switch trial (because they alternated regularly with same trials). This did not eliminate the costs associated with switch trials, indicating that intertrial priming effects could not be overcome by top-down settings. A recent study by Theeuwes, Reimann, and Mortier (in press) offers further support for the automatic priming account. In one of their visual search experiments, participants were required to look for either a shape- or a color-defined target (with target types randomly mixed). The search displays were preceded by a cue, which consisted of one of the two target types (i.e., the actual target shape or color). The cue was valid on $80 \%$ of the trials. The results showed that search was speeded on valid trials relative to invalid trials, suggesting that the observers made active use of the cue. However, a subsequent experiment indicated otherwise. In this experiment, the cue was valid on only $16.6 \%$ of the trials. That is, a shape cue was now actually predictive of a color target (and vice versa, a color cue implied that a shape target would be most likely). Still, the benefit for valid trials was virtually the same as in the first experiment. This indicates that search was driven more by the just previously processed stimulus (i.e., the cue) than by what this stimulus actually meant-in line with an automatic priming account.

\section{Priming and Competition}

Interestingly, we also found an interaction between intertrial relationship and singleton distractor presence in Experiment 2, where target and singleton distractors always had different shapes and, thus, priming could operate only between the targets themselves. Apparently, singleton distractor presence enhances the role of intertrial priming even when the singleton distractor itself is not primed. To account for this finding, we start from Müller et al.'s (1995) idea that intertrial effects are caused by weight shifting. Features associated with the target receive more weight, whereas features associated with the distractor lose weight. What we propose here is that when there is more competition for attention (e.g., when a singleton distractor is present), larger weights will need to be assigned to features associated with the target for it to win this competition. The larger weight results in stronger intertrial priming effects, measurable on the subsequent trial. This way, intertrial priming may be argued to be an automatic adjustment mechanism, contingent upon task requirements such as the need for competitive selection (cf. Olivers \& Humphreys, 2003; Wolfe, Butcher, Lee, \& Hyle, 2003). We would therefore like to propose that intertrial priming is not a strict top-down process (in the sense that it is under the voluntary control of the observer), but neither is it a strict bottom-up process (in the sense that it is fully driven by the current stimulus). Instead, it lies somewhere in between, automatically adapting the organism to a changing environment.

The present manipulations and results bear some similarities to the negative priming paradigm, in which a distractor presented on one trial becomes the target on the next. The typical finding is that processing of this target is delayed, relative to targets that are unrelated to previous trials (e.g., Tipper, 1985). Tipper has suggested that, in order to select the target, the distractor is inhibited and that this inhibition automatically carries over to the next trial - a mechanism that would fit with our present proposal of automatic competition-driven priming effects between targets and distractors (see Experiment 1). However, more recent theories have offered alternative explanations of negative priming (for overviews, see, e.g., Milliken, Joordens, Merikle, \& Seiffert, 1998; Neill, Valdes, Terry, \& Gorfein, 1992; Tipper, 2001). Note further that we also found priming effects in Experiment 2 , in which, in contrast to the negative priming task, the target and the singleton distractor did not switch identity (and thus, only between-target priming could occur).

\section{Features or Dimensions}

In our experiments, priming occurred on the feature level (i.e., the specific target shape). This is in accordance with Maljkovic and Nakayama (1994), who also found that intertrial priming influenced attentional processes on a feature level (in their case, color). However, Müller et al. (1995) have found that intertrial effects operate mainly on a dimension level, rather than on a feature level (see also Olivers \& Humphreys, 2003, who found evidence for dimension-priming, together with small feature-priming, effects). The difference between our findings and those in Müller et al. might be the result of their participants' 
performing a cross-dimension search task, whereas our participants performed a within-dimension search task. We speculate that in a cross-dimension search task, priming operates on a dimension level (since this is the level on which the competition takes place), whereas in a withindimension search task, intertrial priming takes place on a feature level. If this indeed turns out to be the case, it would again suggest that intertrial priming mechanisms are adjusted on the basis of task requirements.

The task dependency of intertrial priming is further suggested by Kumada (2001), who found that intertrial priming played a role in a standard present/absent search task, but not in a compound search task. Kumada argued that intertrial priming does not influence selective attention but plays a role at the response level (see Mortier, Theeuwes, $\&$ Starreveld, 2005, for similar issues). However, note that we, too, had participants perform a compound search task but, contrary to Kumada, still found an effect on RTs as a result of intertrial priming. Also Maljkovic and Nakayama (1994, 2000), as well as Olivers and Humphreys (2003), found that intertrial priming affected compound search. Together with its effects on singleton distractor interference (as shown in the present study), this suggests that intertrial priming does affect attentional processes after all and not just response-related processes.

\section{Target Uncertainty}

Regardless of any singleton distractor effects, RTs in the mixed condition were slower overall than those in the pure condition. Because this slowing was not affected by the presence of a singleton distractor, it is unlikely that it was caused by a general widening of the attentional selection process. Instead, the overall character of the slowing suggests that additional processing after selection is responsible. One such process may be the comparison of the selected item with a target template in visual shortterm memory (VSTM; Duncan \& Humphreys, 1989; Hodsoll \& Humphreys, 2001). When a target template is extended to include more target types, matching of the items in VSTM to the target template takes more time (cf. Sternberg, 1969).

\section{Conclusion}

Two separate mechanisms appear to play a role in visual search when the target is uncertain. One mechanism, presumably involving the matching of the target, causes RTs to be longer overall but does not influence the effect of a singleton distractor on selective attention. The other mechanism, intertrial priming, does influence selective attention by differentially (de)activating the target and possible distractors. However, target uncertainty does not change attentional capture by irrelevant singletons beyond the influence of preceding trials. Importantly, a lack of knowledge of the target does not cause participants to be more distracted by irrelevant yet salient objects.

\section{REFERENCES}

BACON, W. F., \& Egeth, H. E. (1994). Overriding stimulus-driven attentional capture. Perception \& Psychophysics, 5, 485-496.

Duncan, J., \& Humphreys, G. W. (1989). Visual search and stimulus similarity. Psychological Review, 96, 433-458.

Folk, C., Remington, R. W., \& Johnston, J. C. (1992). Involuntary covert orienting is contingent on attentional control setting. Journal of Experimental Psychology: Human Perception \& Performance, 18, 1030-1044.

Hillstrom, A. P. (2000). Repetition effects in visual search. Perception \& Psychophysics, 62, 800-817.

Hodsoll, J., \& Humphreys, G. W. (2001). Driving attention with the top down: The relative contribution of target templates to the linear separability effect in the size dimension. Perception \& Psychophysics, 63, 918-926.

Kumada, T. (2001). Feature-based control of attention: Evidence for two forms of dimension weighting. Perception \& Psychophysics, 63, 698-708.

Maljkovic, V., \& Nakayama, K. (1994). Priming of pop-out: I. Role of features. Memory \& Cognition, 22, 657-672.

Maljkovic, V., \& Nakayama, K. (2000). Priming of pop-out: III. A short-term implicit memory system beneficial for rapid target selection. Visual Cognition, 7, 571-595.

Milliken, B., Joordens, S., Merikle, P. M., \& Seiffert, A. E. (1998). Selective attention: A reevaluation of the implications of negative priming. Psychological Review, 105, 203-229.

Mortier, K., Theeuwes, J., \& Starreveld, P. (2005). Response selection modulates visual search within and across dimensions. Journal of Experimental Psychology: Human Perception \& Performance, 31, 542-557.

Müller, H. J., Heller, D., \& Ziegler, J. (1995). Visual search for singleton feature targets within and across feature dimensions. Perception \& Psychophysics, 57, 1-17.

Neill, W. T., Valdes, L. A., Terry, K. M., \& Gorfein, D. S. (1992). Persistence of negative priming: II. Evidence for episodic trace retrieval. Journal of Experimental Psychology: Learning, Memory, \& Cognition, 18, 993-1000.

Olivers, C. N. L., \& Humphreys, G. W. (2003). Attentional guidance by salient feature singletons depends on intertrial contingencies. Journal of Experimental Psychology: Human Perception \& Performance, 29, 650-657.

PAshler, H. (1988). Cross-dimensional interaction and texture segregation. Perception \& Psychophysics, 43, 307-318.

SternberG, S. (1969). The discovery of processing stages: Extensions of Donders' method. In W. G. Koster (Ed.), Attention and performance II (pp. 276-315). Amsterdam: North-Holland.

Theeuwes, J. (1991). Cross-dimensional perceptual selectivity. Perception \& Psychophysics, 50, 184-193.

Theeuwes, J. (1992). Perceptual selectivity for color and form. Perception \& Psychophysics, 51, 599-606.

Theeuwes, J., Reimann, B., \& Mortier, K. (in press). Visual search for featural singletons: No top-down modulation, only bottom-up priming. Visual Cognition.

TIPPER, S. P. (1985). The negative priming effect: Inhibitory priming by ignored objects. Quarterly Journal of Experimental Psychology, 37A, 571-590.

TIPPER, S. P. (2001). Does negative priming reflect inhibitory mechanisms? A review and integration of conflicting views. Quarterly Journal of Experimental Psychology, 54A, 321-343.

Wolfe, J. M., Butcher, S. J., Lee, C., \& Hyle, M. (2003). Changing your mind: On the contributions of top-down and bottom-up guidance in visual search for feature singletons. Journal of Experimental Psychology: Human Perception \& Performance, 29, 483-502.

YANTIS, S., \& EgETH, H. E. (1999). On the distinction between visual salience and stimulus-driven attentional capture. Journal of Experimental Psychology: Human Perception \& Performance, 25, 661-676. 